\title{
Radiation-Induced Heterogeneous Polymerization. I. Kinetic Study of Bulk Polymerization of Acrylonitrile
}

\author{
Takeshi Wada, Terutaka Watanabe, \\ and Masaaki TAKEHISA \\ Japan Atomic Energy Research Institute, Takasaki \\ Radiation Chemistry Research Establishment, \\ Takasaki, Gunma, Japan.
}

(Received February 28, 1972)

\begin{abstract}
The radiation-induced bulk polymerization of acrylonitrile at $20^{\circ} \mathrm{C}$ was studied kinetically. The polymerization proceeded with precipitation of polymer from the liquid monomer and reached a steady state after an initial acceleration period. The dose rate exponent of the rate of polymerization at steady state was about 0.9. On the basis of the kinetic treatment of the polymerization which contained both firstand second-order terminations for the concentration of progagating radical, the rate constant of each elementary reaction was found to be: $k_{\mathrm{i}}=2.90 \times 10^{-11}, k_{\mathrm{p}}=2.40, k_{\mathrm{tr}}=$ $1.48 \times 10^{-4}, k_{\mathrm{t} 1}=6.33 \times 10^{-4}, k_{\mathrm{t} 2}=2.27 \times 10^{2}$.

KEY WORDS Heterogeneous Polymerization / Radiation Polymerization / Kinetics / Acrylonitrile / Rate Constant /
\end{abstract}

There have been several reported papers on the radiation-induced polymerization of acrylonitrile in bulk in which the polymer precipitated from the liquid monomer. For this heterogeneous polymerization it is known that the dose rate exponent of the rate of polymerization lies between 0.5 and 1.0 , and there is an acceleration period in the earlier stages of the reaction. ${ }^{1-4}$

It has also been reported that, in the heterogeneous polymerization of acrylonitrile with use of radical initiators, the initiator concentration exponent and the existence of an acceleration period were similar to those in the radiation induced polymerization. ${ }^{5-8}$

Bamford, et al., have explained the difference between the initiator-concentration exponent in homogeneous polymerization and that in heterogeneous polymerization by considering a unimolecular termination by occlusion of propagating radical in a clump of polymer molecules. Evidence for the existence of this radical has been discovered by a reaction with $\alpha, \alpha$-diphenyl$\beta$-picryl hydrazyl ${ }^{9}$ and by ESR techniques. ${ }^{10-11}$

Kinetic treatment in the steady state with the use of radical initiators in bulk ${ }^{6}$ and in benzene ${ }^{7}$ was carried out based on the mechanism in which a first-order termination by radical occlusion and a second-order temination by radical recombination take place simultaneously. However, the rate constant for each elementary reaction in these heterogeneous polymerizations has seldom been determined.

Taking information from the acceleration period in the earlier stages, a kinetic treatment to determine the rate constants for the radiationinduced polymerization of ethylene (which is a heterogeneous polymerization and contains both first- and second-order terminations) has been reported elsewhere. ${ }^{12}$

In this paper, based on information from the acceleration period, the results of radiation induced polymerization of acrylonitrile in bulk at $20^{\circ} \mathrm{C}$ and the kinetic treatment derived based on a reaction mechanism containing both firstand second-order terminations ${ }^{7}$ are reported. Using the present kinetic treatment, the rate constant for each elementary reaction is determined from the relationship between the amount of polymerized monomer and the number of polymer chains and the reaction time. 


\section{EXPERIMENTAL}

Acrylonitrile obtained from Kanto Chemical Co. Inc., was used for the polymerization after distillation. Aliquot of distilled acrylonitrile pipetted in a glass ampoule, was degassed five times by repeated freezing-pumping-thawing cycles using a vacuum line and sealed off under vacuum. The polymerization was carried out in a temperature-controlled bath using cobalt 60 or cesium 137 as a $\gamma$-radiation source. After the irradiation, the polymerization was stopped by placing the ampoule in liquid nitrogen. The polymer obtained was separated from the monomer with a glass filter, washed well with acetone on the filter, dried at $80^{\circ} \mathrm{C}$ for $24 \mathrm{hr}$ and weighed.

The intrinsic viscosity of the polymer in dimethyl formamide at $35^{\circ} \mathrm{C}$ was measured. The molecular weight of polymer was determined from the intrinsic viscosity by use of equation (1), which was itself determined by measurement of the osmotic pressure for polymer samples produced by radiation polymerization. ${ }^{13}$

$$
[\eta]=2.10 \bar{M}_{n}^{0.625}
$$

\section{RESULTS AND DISCUSSION}

Figure 1 shows the time-conversion curves at various dose rates. It was found that the rate of polymerization increased with time in the earlier stages of the reaction, but, after this initial acceleration, the curves became virtually straight lines, indicating the existence of a steady state. The steady state was reached earlier at higher dose rates. This initial acceleration has been observed in the heterogeneous polymerization of acrylonitrile by radical initiators. ${ }^{5-8}$ Bamford, et al. ${ }^{5}$ have shown, on the basis of the results from polymerization of the monomer recovered by distillation from polymerized system, that the acceleration period in the earlier stages was not an induction period. Therefore, it is considered that the acceleration is due to the accumulation of the propagating radical.

The rate of polymerization at steady state $R_{\mathrm{S}}$ was plotted against the dose rate in Figure 2. As shown in this figure, the rate of polymerization obeys the relationship

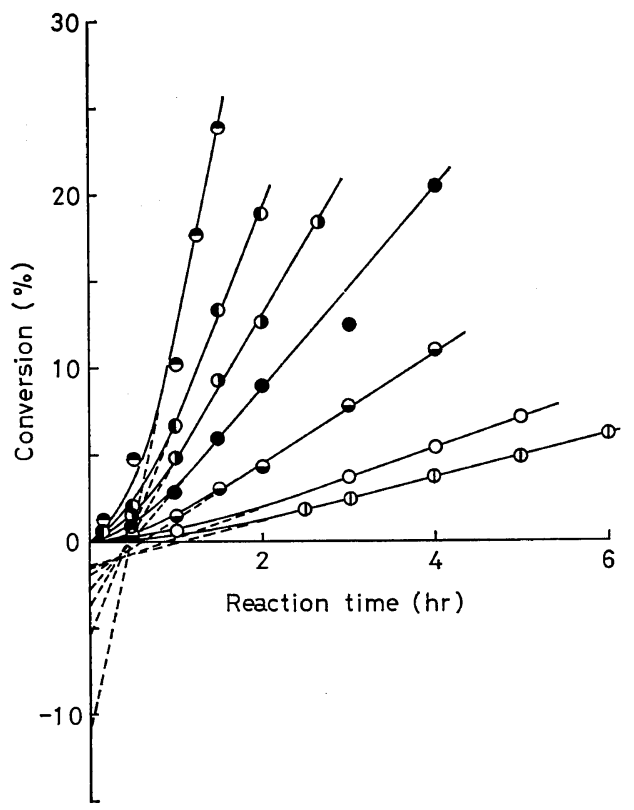

Figure 1. Time-conversion curves in radiationinduced bulk polymerization of acrylonitrile at $20^{\circ} \mathrm{C}$. Dose rate $\times 10^{-4}(\mathrm{rad} / \mathrm{hr}): \Theta, 15.0 ; 0,7.0$; () $4.8 ; \bullet, 3.0 ; \ominus, 1.6 ; \bigcirc, 0.68 ;(1), 0.46$.

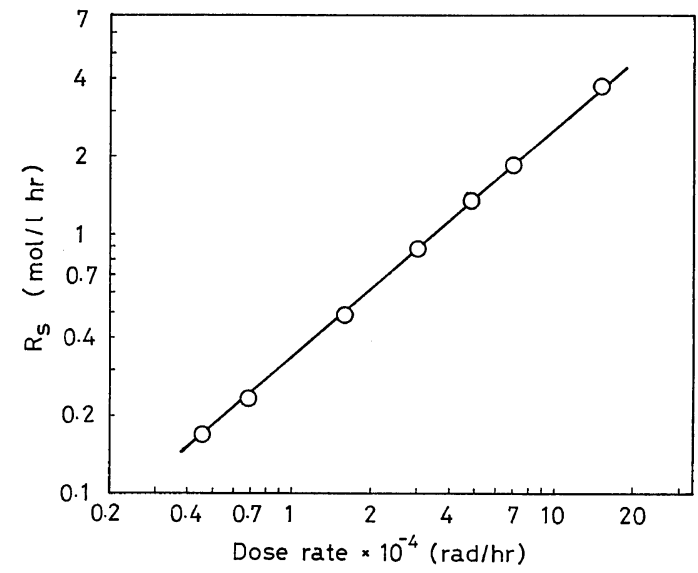

Figure 2. Effect of dose rate on the rate of polymerization at steady state.

$$
R_{\mathrm{S}} \propto I^{0.9}
$$

where $I$ is dose rate.

Prevot-Bernas and Sebban-Dannon ${ }^{3}$ have reported that the initial and the limiting rates at $20^{\circ} \mathrm{C}$ were as follows.

$$
R \propto I^{0.8}
$$


Berstein $^{1}$, also, has found the dose rate dependency of the conversion at $2680 \mathrm{r}$ was neither 0.5 nor 1.0 .

Such dose rate dependencies different from those in homogeneous polymerization indicate that the termination reaction is composed of neither the first- nor the second-order reaction for the concentration of propagating radical alone, but of both the first- and second-order reactions in bulk polymerization of acrylonitrile.

Figure 3 shows the plot of molecular weight against conversion into polymer. It was found

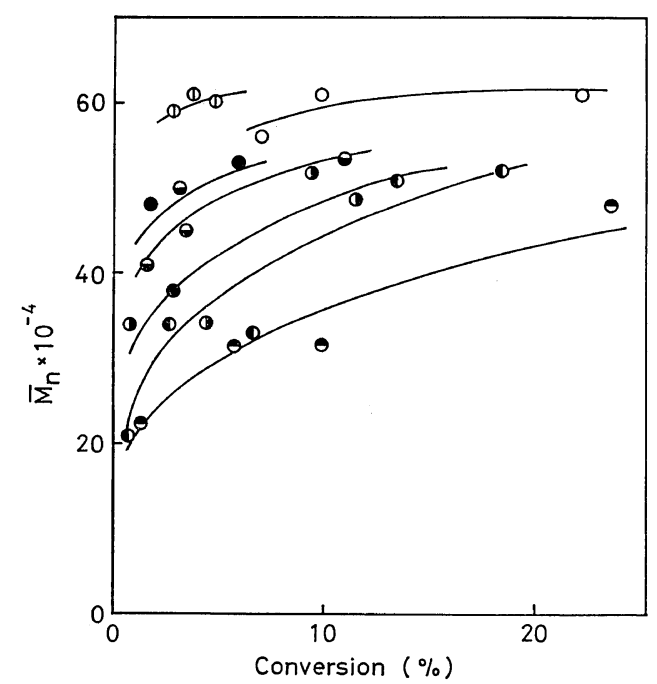

Figure 3. Molecular weight of polymer vs. conversion into polymer at various dose rates. Dose rates and symbols are the same as in Figure 1.

that the molecular weight increased with a decrease in the dose rate. The molecular weight had a tendency to increase gradually in the earlier stages of the reaction and levelled off to a constant value with the progress of the reaction.

On the other hand, the number of polymer chains increased almost linearly with the reaction time after an acceleration period in the earlier stages as shown in Figure 4.

On the basis of these results the reaction mechanism was considered to be as follows

Initiation

$$
\begin{aligned}
& \mathrm{M} \longrightarrow \mathrm{R}_{1} \cdot \\
& R_{\mathrm{i}}=k_{\mathrm{i}}[\mathrm{M}] I
\end{aligned}
$$

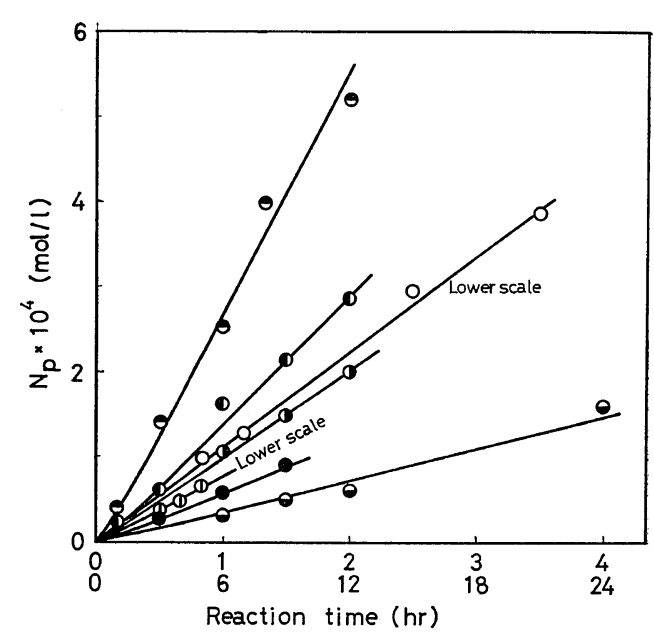

Figure 4. Number of polymer chains $v s$. reaction time at various dose rates. Dose rates and symbols are the same as in Figure 1.

Propagation

$$
\begin{gathered}
\mathbf{R}_{n} \cdot+\mathrm{M} \longrightarrow \mathrm{R}_{n+1} \cdot \\
R_{\mathrm{p}}=k_{\mathrm{p}}[\mathrm{R} \cdot][\mathrm{M}]
\end{gathered}
$$

Chain transfer to monomer

$$
\begin{gathered}
\mathrm{R}_{n} \cdot+\mathrm{M} \longrightarrow \mathrm{P}_{n}+\mathrm{R}_{1} \cdot \\
\boldsymbol{R}_{\mathrm{tr}}=k_{\mathrm{tr}}[\mathrm{R} \cdot][\mathrm{M}]
\end{gathered}
$$

Chain transfer to polymer

$$
\begin{gathered}
\mathbf{R}_{n} \cdot+\mathrm{P}_{m} \longrightarrow \mathbf{P}_{n}+\mathbf{R}_{m} . \\
\boldsymbol{R}_{\mathrm{trp}}=k_{\mathrm{trp}}[\mathrm{R} \cdot][\mathrm{P}]
\end{gathered}
$$

First-order termination

$$
\begin{aligned}
\mathbf{R}_{n} \cdot \longrightarrow \mathrm{P}_{n} \\
\boldsymbol{R}_{\mathrm{t} 1}=k_{\mathrm{t} 1}[\mathrm{R} \cdot]
\end{aligned}
$$

Second-order termination

$$
\begin{gathered}
\mathbf{R}_{m} \cdot+\mathbf{R}_{n} \cdot \longrightarrow \mathbf{P}_{m+n} \\
\boldsymbol{R}_{\mathrm{t} 2}=k_{\mathrm{t} 2}[\mathbf{R} \cdot]^{2}
\end{gathered}
$$

where $\mathbf{M}$ is monomer, $\mathbf{R}_{n}$. is propagating radical composed of $n$-monomers and $\mathrm{P}_{n}$ is polymer composed of $n$-monomers. $R_{\mathrm{i}}, R_{\mathrm{p}}, R_{\mathrm{tr}}, R_{\mathrm{trp}}$, $R_{\mathrm{t} 1}$, and $R_{\mathrm{t} 2}$ are the rates of initiation, propagation, chain transfer to monomer, chain transfer to polymer, first-order termination, and secondorder termination, $k_{\mathrm{i}}, k_{\mathrm{p}}, k_{\mathrm{tr}}, k_{\mathrm{trp}}, k_{\mathrm{t} 1}$, and $k_{\mathrm{t} 2}$ are the corresponding rate constants. 
As previously described, ${ }^{12}$ the following relationships were found among the rate of polymerization at a steady state $R_{\mathrm{s}}$, the ordinate intercept obtained by extrapolating the linear portions at the steady state in the time-conversion curve $b$, the rate of increase in the number of polymer chains at steady state $e$, and the dose rate $I$ on the basis of the reaction mechanism described above

$$
R_{\mathrm{s}}=c\left(\frac{I}{R_{\mathrm{s}}}\right)-d
$$

or

$$
\begin{gathered}
\frac{I}{R_{\mathrm{s}}{ }^{2}}=\left(\frac{1}{c}\right)\left(\frac{d}{R_{\mathrm{s}}}+1\right) \\
b=\left(\frac{k_{\mathrm{p}}[\mathrm{M}]}{k_{\mathrm{t} 2}}\right) \ln \left(\frac{2 \sqrt{1+4 c I / d^{2}}}{1+\sqrt{1+4 c I / d^{2}}}\right) \\
\frac{e}{I}=g\left(\frac{R_{\mathrm{s}}}{I}\right)+h
\end{gathered}
$$

or

$$
\frac{e}{R_{\mathrm{s}}}=h\left(\frac{I}{R_{\mathrm{s}}}\right)+g
$$

where

$$
\begin{gathered}
c=\frac{k_{\mathrm{p}}{ }^{2} k_{\mathrm{i}}[\mathrm{M}]^{3}}{k_{\mathrm{t} 2}} \\
d=\frac{k_{\mathrm{p}} k_{\mathrm{t} 1}[\mathrm{M}]}{k_{\mathrm{t} 2}} \\
g=\frac{2 k_{\mathrm{tr}}[\mathrm{M}]+k_{\mathrm{t} 1}}{2 k_{\mathrm{p}}[\mathrm{M}]} \\
h=\frac{k_{\mathrm{i}}[\mathrm{M}]}{2}
\end{gathered}
$$

The plot of eq 16 using the data from this experiment failed to produce a straight line (as shown in Figure 5). These termination reactions were therefore not simple competitive reactions.

On the other hand, Imoto, et al. ${ }^{7}$, found that the rate of polymerization at steady state was well explained kinetically by assuming that the ratio of the radicals terminated by first-order reaction to those terminated by second-order reaction was a constant depending on reaction conditions but independent of reaction time. However, the relationships between $b, e, f$, and $I$ have not been discussed. In this report, a further development of the kinetic treatment

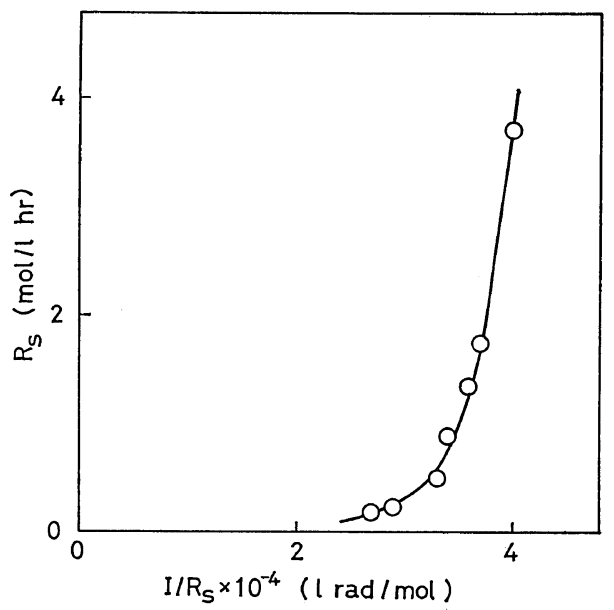

Figure 5. Plots of $R_{\mathrm{S}} v s$. $I / R_{\mathrm{s}}$.

was considered on the basis of the same assumption for the termination scheme.

When the fractions of the propagating radicals terminated by first- and second-order reactions are shown as $\rho$ and $\sigma$ (or $1-\rho$ ), the concentration of the propagating radicals which are terminated by these reactions, $\left[\mathbf{R}_{1} \cdot\right]$ and $\left[\mathbf{R}_{2} \cdot\right]$, are expressed by eq 24 and 25 , respectively ${ }^{7}$

$$
\begin{aligned}
& \frac{\mathrm{d}\left[\mathrm{R}_{1} \cdot\right]}{\mathrm{d} t}=\rho k_{\mathrm{i}}[\mathrm{M}] I-k_{\mathrm{t} 1}\left[\mathrm{R}_{1} \cdot\right] \\
& \frac{\mathrm{d}\left[\mathrm{R}_{2} \cdot\right]}{\mathrm{d} t}=\sigma k_{\mathrm{i}}[\mathrm{M}] I-k_{\mathrm{t} 2}\left[\mathrm{R}_{2} \cdot\right]^{2}
\end{aligned}
$$

where

$$
\left[\mathbf{R}_{1} \cdot\right]+\left[\mathbf{R}_{2} \cdot\right]=[\mathbf{R} \cdot]
$$

Integration of eq 24 and 25 gives the concentration of the propagating radical as follows

$$
[\mathrm{R} \cdot]=A\left\{1-\exp \left(-k_{\mathrm{t} 1} t\right)\right\}+B \tanh \left(B k_{\mathrm{t} 2} t\right)
$$
where

$$
\begin{aligned}
& A=\frac{\rho k_{\mathrm{i}}[\mathrm{M}] I}{k_{\mathrm{t} 1}} \\
& B=\sqrt{\frac{\sigma k_{\mathrm{i}}[\mathrm{M}] I}{k_{\mathrm{t} 2}}}
\end{aligned}
$$

Accordingly, the amount of polymerized monomer and number of polymer chain are given by

$$
\begin{aligned}
M_{\mathrm{p}} & =\int_{0}^{t} k_{\mathrm{p}}[\mathrm{R} \cdot][\mathrm{M}] \mathrm{d} t \\
& =k_{\mathrm{p}}[\mathrm{M}]\left[A\left(t-\frac{1-\exp \left(-k_{\mathrm{t} 1} t\right)}{k_{\mathrm{t} 1}}\right)+B t\right.
\end{aligned}
$$


T. Wada, T. Watanabe, and M. Takehisa

$$
\begin{aligned}
& \left.+\frac{\ln \left[\left\{1+\exp \left(-2 B k_{\mathrm{t} 2} t\right)\right\} / 2\right]}{k_{\mathrm{t} 2}}\right] \\
& N_{\mathrm{p}}=\left(k_{\mathrm{i}}[\mathrm{M}] I+k_{\mathrm{tr}}[\mathrm{R} \cdot][\mathrm{M}]-\frac{k_{\mathrm{t} 2}\left[\mathrm{R}_{2} \cdot\right]^{2}}{2}\right) \mathrm{d} t \\
& =k_{1}[\mathrm{M}] I\left(1-\frac{\sigma}{2}\right) t \\
& +k_{\mathrm{tr}}[\mathrm{M}] A\left(t-\frac{1-\exp \left(-k_{\mathrm{t} 1} t\right)}{k_{\mathrm{t} 1}}\right) \\
& +B\left(k_{\mathrm{tr}}[\mathrm{M}] t+\frac{\tanh \left(B k_{\mathrm{t} 2} t\right)}{2}\right) \\
& +\frac{k_{\mathrm{tr}}[\mathrm{M}] \ln \left[\left\{1+\exp \left(-2 B k_{\mathrm{t} 2} t\right)\right\} / 2\right]}{k_{\mathrm{t} 2}}
\end{aligned}
$$

$$
s=\frac{\left(k_{\mathrm{i}}[\mathrm{M}]\right)^{0.5}}{2 k_{\mathrm{t} 2}^{0.5}}
$$

The values of $R_{\mathrm{s}}, b, e$, and $f$ at various dose rates are given by the plots of $M_{\mathrm{p}}$ against $t$ and $N_{p}$ against $t$ based on eq 32 and 33 .

The plots of $R_{\mathrm{S}} / I$ against $I^{-0.5}$ gave a straight line as shown in Figure 6. The slope and the intercept of this straight line gave $m=0.93 \times 10^{-3}$ $\mathrm{mol} \mathrm{rad}^{0.5} / l \mathrm{hr}^{0.5}$ and $n=2.35 \times 10^{-5} \mathrm{~mol} / l \mathrm{rad}$ on the basis of eq $34^{\prime}$.

When the steady state is reached

$$
\begin{gathered}
M_{\mathrm{p}}=R_{\mathrm{s}} t-b \\
N_{\mathrm{p}}=e t-f
\end{gathered}
$$

where

$$
\begin{gathered}
R_{\mathrm{s}}=m I^{0.5}+n I \\
b=p I+q \\
e=C_{\mathrm{m}} R_{\mathrm{s}}+r I \\
f=-C_{\mathrm{m}} b+s I^{0.5}
\end{gathered}
$$

Rearranging eq 34,36 , and 37 leads to

$$
\begin{gathered}
\frac{R_{\mathrm{s}}}{I}=m I^{-0.5}+n \\
\frac{e}{I}=C_{\mathrm{m}}\left(\frac{R_{\mathrm{s}}}{I}\right)+r \\
f I^{-0.5}=-C_{\mathrm{m}} b I^{-0.5}+s
\end{gathered}
$$

where

$$
\begin{gathered}
m=\frac{k_{\mathrm{p}}\left(\sigma k_{\mathrm{i}}\right)^{0.5}[\mathrm{M}]^{1.5}}{k_{\mathrm{t} 2}^{0.5}} \\
n=\frac{\rho k_{\mathrm{i}} k_{\mathrm{p}}[\mathrm{M}]^{2}}{k_{\mathrm{t} 1}} \\
p=\frac{\rho k_{\mathrm{i}} k_{\mathrm{p}}[\mathrm{M}]^{2}}{k_{\mathrm{t} 1}^{2}} \\
q=\frac{k_{\mathrm{p}}[\mathrm{M}] \ln 2}{k_{\mathrm{t} 2}} \\
C_{\mathrm{m}}=\frac{k_{\mathrm{tr}}}{k_{\mathrm{p}}} \\
r=k_{\mathrm{i}}[\mathrm{M}]\left(1-\frac{\sigma}{2}\right)
\end{gathered}
$$

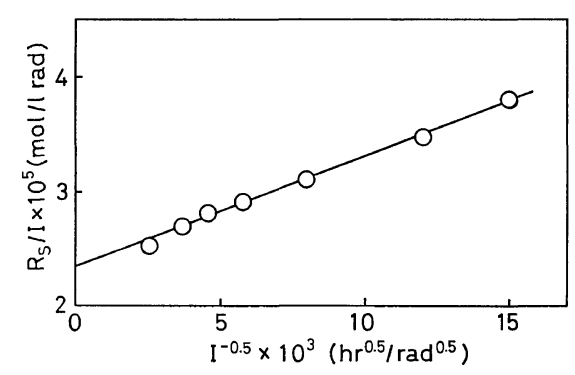

Figure 6. Plots of $R_{\mathrm{S}} / I$ vs. $I^{-0.5}$ on the basis of eq $34^{\prime}$.

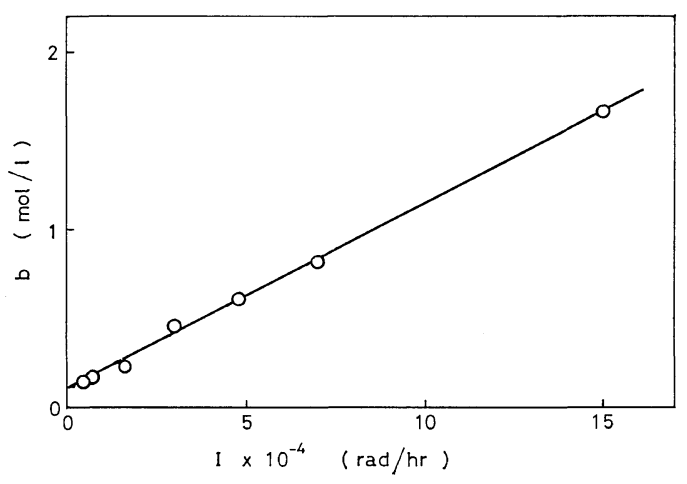

Figure 7. Plots of $b v s$. $I$ on the basis of eq 35 .

As shown in Figure 7, the plots of $b$ against $I$ also produced a straight line and values of $p=1.03 \times 10^{-5} \mathrm{~mol} \mathrm{hr} / l \mathrm{rad}$ and $q=0.11 \mathrm{~mol} / l$ were given by this line.

Furthermore, from the plots of $e / I$ against $R_{\mathrm{s}} / I$ (as shown in Figure 8), the values of $C_{\mathrm{m}}=$ $6.2 \times 10^{-5}$ and $r=0.42 \times 10^{-9} \mathrm{~mol} / l \mathrm{rad}$ were obtained on the basis of the relationship of eq $36^{\prime}$.

On the other hand, as the value of $f$ obtained from Figure 4 was less reliable due to the experimental error in the molecular-weight mesure- 


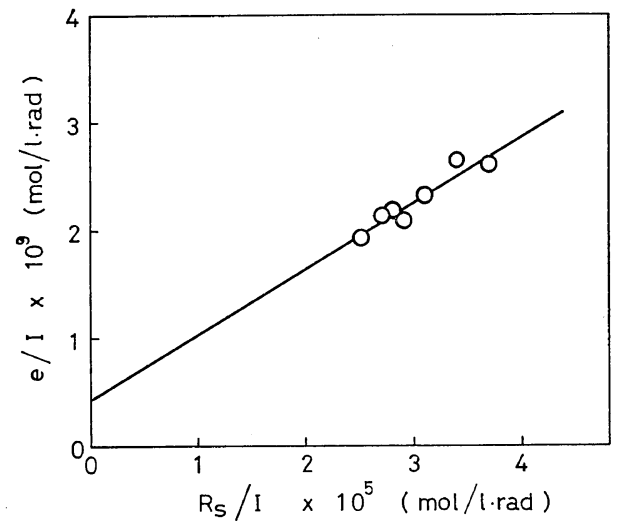

Figure 8. Plots of $e / I$ vs. $R_{\mathrm{S}} / I$ on the basis of eq $36^{\prime}$.

ment, the plot of the relationship of eq $37^{\prime}$ was therefore not shown.

On the basis of the kinetic treatment as described above, the individual rate constants (except $k_{\text {trp }}$ ) can be determined by calculation from these constants obtained from Figures 6, 7, and 8 . The individual rate constants and the values of $\rho$ and $\sigma$ are summarized in Table I.

Table I. Rate constant for each elementary reaction

\begin{tabular}{ccccccc}
\hline $\begin{array}{c}k_{\mathrm{i}}, \\
\mathrm{rad}^{-1}\end{array}$ & $\begin{array}{c}k / \mathrm{mol}, \\
\mathrm{sec}\end{array}$ & $\begin{array}{c}k_{\mathrm{tr}}, \\
\mathrm{sec}\end{array}$ & $\begin{array}{c}k_{\mathrm{t} 1}, \\
\mathrm{sec}^{-1}\end{array}$ & $\begin{array}{r}k_{\mathrm{t} 2}, \\
\mathrm{sec} \\
\mathrm{sec}\end{array}$ & $\rho$ & $\sigma$ \\
\hline $\begin{array}{r}2.90 \times \\
10^{-11}\end{array}$ & 2.40 & $\begin{array}{r}1.48 \times \\
10^{-4}\end{array}$ & $\begin{array}{r}6.33 \times \\
10^{-4}\end{array}$ & $\begin{array}{r}2.27 \times \\
10^{2}\end{array}$ & 0.907 & 0.093 \\
\hline
\end{tabular}

The values of $f$, calculated by using the values of $b, I$, and rate constants, were of the order of $10^{-5} \mathrm{~mol} / l$, which were consistent, within the limits of experimental error, with the result shown in Figure 4.

$G_{\mathrm{r}}$ value of the monomer was found to be 0.53 on the basis of $k_{\mathrm{i}}=2.90 \times 10^{-11} \mathrm{rad}^{-1}$. Bensasson $^{14}$ obtained the $G_{\mathrm{r}}$ value as $5.0 \pm 0.3$ by the reaction with $\alpha, \alpha$-diphenyl- $\beta$-picryl hydrazyl. However this $G_{\mathrm{r}}$ value appears to be very much larger than expected, considering the highly conjugated structure of the acrylonitrile molecule.

Both values of $k_{\mathrm{p}}$ and $k_{\mathrm{t} 2}$ are much smaller than those obtained by Bamford, et al., ${ }^{15}$ namely 1960 and $7.83 \times 10^{8}$ at $60^{\circ} \mathrm{C}$ for the homogeneous polymerization in dimethyl formamide. The substantial differences between the values of $k_{\mathrm{p}}$ and $k_{\mathrm{t} 2}$ for heterogeneous and homogeneous systems must be due to differences in the environment of the propagating radical. This seems to be because the mobility of the propagating radical in the heterogeneous polymerization seems to be much less than in the homogeneous one and because the steric hindrance due to the cluster of the partially crystallized polymer in the heterogeneous polymerization is much larger than in the homogeneous equivalent.

The present value for the transfer constant to monomer was found to be $6.2 \times 10^{-5}$, which agrees with that obtained by other authors (of the order of $10^{-4}$ to $10^{-6}, 3,8,10,16-18$ ) and means that the effect of physical factors such as the mobility of the propagating radical and the steric hindrance of the solid polymer on the chain-transfer reaction is similar to the effect on the propagation reaction.

Moreover the concentration of the radical terminated by the first-order reaction is about 10 times larger than that by the second-order reaction.

Table II. Rates of first- and second-order terminations calcuated from the constants shown in Table I

\begin{tabular}{ccccc}
\hline $\begin{array}{c}I, \\
\mathrm{rad} / \mathrm{hr}\end{array}$ & $\begin{array}{c}{[\mathrm{R} \cdot]_{\mathrm{s}},} \\
\mathrm{mol} / l\end{array}$ & $\begin{array}{c}R_{\mathrm{t} 1}, \\
\mathrm{~mol} / l \mathrm{sec}\end{array}$ & $\begin{array}{c}\boldsymbol{R}_{\mathrm{t} 2,}, \\
\mathrm{~mol} / l \mathrm{sec}\end{array}$ & $R_{\mathrm{t} 1 / R_{\mathrm{t} 2}}$ \\
\hline $10^{3}$ & $4.00 \times 10^{-7}$ & $2.29 \times 10^{-10}$ & $3.13 \times 10^{-13}$ & 732 \\
$10^{4}$ & $2.47 \times 10^{-6}$ & $1.42 \times 10^{-9}$ & $1.19 \times 10^{-11}$ & 119 \\
$10^{5}$ & $1.98 \times 10^{-5}$ & $1.14 \times 10^{-8}$ & $7.70 \times 10^{-10}$ & 14.8 \\
\hline
\end{tabular}

The rates, $R_{\mathrm{t} 1}$ and $R_{\mathrm{t} 2}$ at the steady state, calculated from the values of $k_{\mathrm{t} 1}, k_{\mathrm{t} 2}, \rho, \sigma$, and $[\mathrm{R} \cdot]_{\mathrm{s}}$ are summarized in Table II where the concentration of the propagating radical at the steady state $[R \cdot]_{\mathrm{S}}$ was given by eq 45 obtained from eq 27.

$$
[\mathrm{R} \cdot]_{\mathrm{s}}=A+B
$$

As shown in this table, it is concluded that the first-order reaction is more important than the second-order termination in this polymerization. However, it was found that the ratio $R_{\mathrm{t} 1} / R_{\mathrm{t} 2}$ decreased with increasing dose rate. 


\section{REFERENCES}

1. I. A. Berstein, E. C. Farmer, W. C. Rothschild, and F. F. Spalding, J. Chem. Phys., 21, 1303 (1953).

2. A. Chapiro and J. Sebban-Dannon, J. Chim. Phys., 54, 776 (1957).

3. A. Prevot-Bernas and J. Sebban-Dannon, ibid., 53, 418 (1957).

4. H. Bernas and M. Bodard, J. Polym. Sci., 48, 167 (1960).

5. C. H. Bamford and A. D. Jenkins, Proc. Roy. Soc. (London), A216, 515 (1953).

6. W. M. Thomas and J. J. Pellon, J. Polym. Sci., 13, 329 (1954).

7. M. Imoto and H. Takatsugi, Makromol. Chem., 23, 119 (1957).

8. N. Tokura, M. Matsuda, and F. Yazaki, ibid., 42, 108 (1960).
9. C. H. Bamford and A. D. Jenkins, Proc. Roy. Soc. (London), A228, 220 (1955).

10. A. D. Jenkins and C. H. Bamford, J. Polym. Sci., 34, 181 (1959).

11. D. J. E. Ingram, M. C. R. Symons, and M. G. Townsend, Trans. Faraday Soc., 54, 409 (1958).

12. T. Wada, T. Watanabe, and M. Takehisa, $J$. Polym. Sci., Part A-1, 9, 2659 (1971).

13. T. Wada and M. Takehisa, unpublished results.

14. A Chapiro, "Radiation Chemistry of Polymeric Systems," Interscience Publishers, Inc., New York, N. Y., 1961, p 206.

15. C. H. Bamford, A. D. Jenkins, and R. Johnston, Proc. Roy. Soc. (London), A241, 429 (1955).

16. P. F. Onyon, J. Polym. Sci., 22, 19 (1956).

17. H. Kitagawa, Makromol. Chem., 64, 229 (1963).

18. M. Yoshida and K. Tanouchi, ibid., 71, 216 (1964). 